\title{
Sífilis na gravidez
}

\author{
Syphilis in pregnancy
}

\section{Sífilis en el embarazo}

\author{
Alessandra B.A. Damasceno, Denise L. M. Monteiro," Luiza B. Rodrigues, \\ Danielle B. S. Barmpas, Luciane R. P. Cerqueira, Alexandre J. B. Trajano
}

\section{Resumo}

A sífilis é causada pelo Treponema pallidum e transmitida por via sexual, hematogênica ou vertical (TV) durante qualquer período da gravidez. Por orientação do Ministério da Saúde, seu rastreio seu e tratamento são oferecidos de rotina a todas as gestantes que realizam o pré-natal. No entanto, as taxas de morbidade materna, infecção congênita e mortalidade perinatal permanecem altas, representando ainda desafio à saúde pública. A persistência da alta prevalência de sífilis congênita, apesar dos programas específicos de prevenção, é inquietante. Esta revisão visa contribuir para a divulgação do conhecimento atual no país e a elaboração de medidas que possam reduzir a transmissão vertical e a morbimortalidade materno-infantil da sífilis. A prevalência de sífilis em gestantes no Brasil é de 1,6\%. A região Sudeste possui a maior taxa de notificação de sífilis congênita, e o Estado do Rio de Janeiro tem as maiores taxas de incidência por nascidos vivos (9,8/1.000), segundo dados do Ministério da Saúde. O custo da prevenção da infecção congênita, incluindo teste e tratamento, é menor que US\$1,50 por pessoa, e o risco de transmissão vertical na gravidez varia de 70 a 100\%, nos casos de sífilis recente, e 30 a $40 \%$, na sífilis tardia. Nesta revisão, apresentamos uma discussão sobre o diagnóstico e o tratamento precoce da sífilis na gravidez, que, quando realizado adequadamente, pode prevenir resultados gestacionais desfavoráveis para o binômio mãe-filho. Um melhor entendimento da doença permite buscar formas de melhorar as políticas públicas, colaborando com a diminuição da prevalência da sífilis e suas complicações no Brasil.

Descritores: Sífilis; Doenças sexualmente transmissíveis; Prevalência, Gravidez; Transmissão de doença infecciosa; /prevenção \& controle.

\begin{abstract}
Syphilis is caused by the Treponema pallidum and its mode of transmission is sexual, blood borne or from mother-to-child (vertical) throughout the pregnancy. According to the guidance of the brazilian Ministry of Health, serological screening and treatment are routinely offered to all pregnant women attending antenatal care, nevertheless rates of maternal morbidity, congenital infection and perinatal mortality remain high and the disease still represents a challenge for public health. The persistence of the high prevalence of congenital syphilis despite the targeted prevention programs is unsettling. This review aims to contribute to the spread of the current knowledge in our country and the elaboration of measures which can reduce vertical transmission and mother and child morbimortality. The prevalence of syphilis in pregnant women in Brazil is 1.6\%. The Southeast region has the highest rate of congenital syphilis notification, and the State of Rio de Janeiro has the highest incidence per live births $(9.8 / 1,000)$, according to data of the Ministry of Health. The cost of preventing congenital syphilis, testing and therapy included, is lower than U\$1.50 per person and the risk of mother-to-child transmission during pregnancy ranges from 70 to $100 \%$ in cases of recent syphilis to 30 to $40 \%$ in late syphilis. This review presents a discussion about the diagnosis and early treatment of syphilis in pregnancy, which can prevent unfavourable pregnancy outcomes for both mother and child when properly administered. A better understanding of the condition allows the search for improvement in public policies, collaborating to the reduction of the prevalence of syphilis and its complications in Brazil.
\end{abstract}

Keywords: Syphilis; Sexually Transmitted Diseases; Prevalence; Pregnancy; Disease transmission, infectious; / prevention \& control. 


\section{Resumen}

La sífilis es causada por el Treponema pallidum y transmitida por vía sexual, hematógena o vertical (TV) durante cualquier periodo del embarazo. Por orientación del Ministerio de Salud del Brasil, su detección y tratamiento son ofrecidos por rutina a todos las gestantes que realizan el prenatal. Sin embargo, las tasas de morbilidad materna, infección congénita y mortalidad perinatal permanecen altas, representando todavía un desafío para la salud pública. La persistencia de la alta prevalencia de sífilis en gestantes en el Brasil es del 1,6\%. La región sudeste posee la mayor tasa de notificación de sífilis congénita, y el Estado de Rio de Janeiro tiene las mayores incidencias por nacidos vivos (9,8/1.000), según datos del Ministerio de Salud. El costo de la prevención congénita, incluyendo test y tratamiento, es menor de U\$1,50 por persona, y el riesgo de transmisión vertical en el embarazo varia del 70 al 100\%, en los casos de sífilis reciente, y 30 al 40\%, en la sífilis tardía. En esta revisión presentamos una discusión sobre el diagnóstico y el tratamiento precoz de la sífilis en el embarazo que, cuando es realizado adecuadamente puede prevenir resultados gestacionales desfavorables para el binomio madre-hijo. Un mejor entendimiento de la enfermedad permite buscar formas de mejorar las políticas públicas, colaborando con la disminución de la prevalencia de la sífilis y sus complicaciones en el Brasil.

Palabras clave: Sífilis; Enfermedades sexualmente transmisibles; Prevalencia, Embarazo; Transmisión de enfermedad infecciosa; /prevención y control.

\section{Introdução}

A sífilis, doença sexualmente transmitida causada pela bactéria Treponema pallidum, se apresenta como um desafio à saúde pública em todo o mundo. É uma doença transmitida por via sexual (sífilis adquirida) e vertical (sífilis congênita) pela placenta da mãe para o feto. Outras formas de transmissão podem ser por via indireta (objetos contaminados) e por transfusão sanguínea.'

Apesar de possuir agente etiológico bem definido, formas conhecidas de transmissão e tratamentos com excelentes índices de cura, nota-se ainda índices elevados de incidência da doença. Esse fato é a tradução da carência total de aplicação de protocolos, que, se fossem cumpridos, permitiriam a redução da disseminação da doença. ${ }^{2}$

No Brasil, a notificação atinge somente 32\% dos casos de sífilis gestacional e apenas $17,4 \%$ de sífilis congênita. Esses dados reforçam a falta de qualidade nos serviços de assistência pré-natal e para o parto. Como a maioria das gestantes encontra-se na fase latente da sífilis, torna-se necessária a adoção de testes sorológicos para o diagnóstico. ${ }^{3}$

A não realização de pré-natal, a gravidez na adolescência, o uso de drogas ilícitas pela mãe ou pelo parceiro (principalmente crack/cocaína), a ausência de parceiro sexual fixo e/ou a existência de múltiplos parceiros, baixa escolaridade e nível socioeconômico, multiparidade, acesso limitado aos serviços de saúde e presença de outras doen- ças sexualmente transmissíveis (DSTs) na mulher ou no parceiro são fatores de risco associados à infecção congênita. ${ }^{4}$

Na presença de infecção por sífilis, o rastreio para afastar outras DSTs é preconizado. A sífilis aumenta cerca de 10 vezes o risco de infecção pelo HIV (e em 18 vezes na presença de úlceras genitais). Deve-se sempre descartar a possibilidade da coinfecção da sífilis com o HIV pelo risco de transmissão vertical. ${ }^{5,6}$

Segundo o Ministério da Saúde, entre o ano de 1998 e junho de 2012 foram notificados 80.041 casos de sífilis congênita em menores de um ano de idade, sendo $45,9 \%$ desses casos provenientes da região Sudeste, onde o estado do Rio de Janeiro apresentava, em 2011, uma das maiores taxas de incidência ( 9,8 por mil nascidos vivos). Foram avaliados os óbitos notificados por sífilis congênita no mesmo período, evidenciando 1780 casos no país, sendo 758 óbitos provenientes do Estado do Rio de Janeiro, correspondendo a 42\% dos óbitos do Brasil. ${ }^{7}$ A prevalência da sífilis em gestantes no Brasil, segundo o Ministério da Saúde, é de 1,6\%. O custo da prevenção da sífilis congênita é menor que U\$ 1,50 com teste e tratamento por pessoa. ${ }^{8}$

É um contrassenso uma doença de fácil diagnóstico e terapêutica medicamentosa de tão baixo custo apresentar incidência tão elevada na nossa população. As consequências são desastrosas, principalmente para as pacientes em idade reprodutiva, pelos riscos de transmissão vertical nos casos de sífilis congênita. Sendo assim, é de suma importância um estudo de revisão que poderá ser 
útil para melhor entendimento e aplicabilidade de políticas de saúde que ajudariam no combate à doença.

\section{Aspectos clínicos}

A sífilis pode ser classificada como primária, secundária, latente, terciária ou congênita.

A sífilis primária ocorre após três semanas de exposição; há o aparecimento de úlcera, única, medindo entre 0,3 e 3,0 cm, indolor, no local da inoculação, com resolução espontânea em três a seis semanas, podendo ocasionalmente aparecer lesões múltiplas, sendo mais comuns quando associadas à coinfecção pelo HIV. ${ }^{9}$

A sífilis secundária ocorre após seis semanas a seis meses da infecção primária não tratada, manifestando-se como rash cutâneo eritematoso e simétrico em tronco e extremidades, notadamente nas regiões palmar e plantar. Os sintomas gerais mais relatados são mal-estar, cefaleia, febre, prurido (42\%) e hiporexia. Outros, menos comuns, são dor nos olhos, dor óssea, artralgia, meningismo, irite e rouquidão. $\mathrm{O}$ paciente nessa fase é muito contagioso. ${ }^{10}$

A fase latente da doença inclui os pacientes assintomáticos, sem envolvimento sistêmico da doença, com sorologia positiva. Pode ser precoce (menos de um ano de evolução) ou tardia (mais de um ano de evolução).

A sífilis terciária ocorre em 1 a 10 anos, com casos de até 50 anos para que a evolução se manifeste. É caracterizada pela formação de gomas sifilíticas, que podem acometer principalmente pele, mucosas, esqueleto. As manifestações mais graves da sífilis terciária são o acometimento neurológico (paralisia geral progressiva, pupilas de Argyll Robertson, tabes dorsalis) e cardiovascular (aortite, aneurisma de aorta, regurgitação aórtica). Aproximadamente $40 \%$ dos pacientes com complicações cardiovasculares possuem acometimento neurológico associado."

A sífilis congênita é adquirida usualmente pelo feto no útero materno, quando o Treponema atravessa a barreira transplacentária, apesar de excepcionalmente poder ser transmitida no nascimento. $^{12,13}$

O risco de transmissão na gestação varia de acordo com o estágio da infecção materna e da idade gestacional em que ocorre a exposição fetal, podendo chegar de 70 a $100 \%$ nos casos de sífilis recente e 30 a $40 \%$ nos casos de sífilis tardia. ${ }^{14}$

O quadro clínico do recém-nato variará de acordo com a fase da gestação em que a infecção tiver ocorrido. Quando a infecção se instalar no último trimestre, a criança apresentará maior probabilidade de nascer assintomática. A sífilis congênita pode se apresentar com quadro clínico variável: desde rinite hemorrágica, erupção eritematopapulosa, placas mucosas, condiloma plano, fissuras periorificiais radiadas, pênfigo sifilítico, microadenopatia e hepatoesplenomegalia, choro intenso e plaquetopenia, entre outras manifestações possíveis na sífilis congênita recente, até ceratite intersticial, tíbia em sabre, gomas, hidrartrose bilateral de Clutton e ainda outras manifestações tardias. Estigmas como os dentes de Hutchinson, nódulos de Parrot no crânio, nariz em sela, fronte olímpica e alterações no exame de fundo de olho, apresentando aspecto em "sal e pimenta", podem estar presentes. ${ }^{15}$

A estimativa da Organização Mundial da Saúde (OMS), principalmente nos países em desenvolvimento, é de mais de dois milhões de gestantes infectadas a cada ano e que quase um milhão de recém-natos possuam o diagnóstico de sífilis congênita. Gestantes com sífilis possuem 12 vezes mais chances de terem algum evento adverso, mesmo após tratamento, do que uma paciente soronegativa. Nas gestantes com sífilis não tratada ou inadequadamente tratada, a taxa de abortamento ou natimortalidade é de $25 \%$, prematuridade ou baixo peso ao nascer, de $13 \%$, neomortalidade, de $11 \%$, e $20 \%$ dos lactentes são sintomáticos. ${ }^{16,17}$

\section{Diagnóstico}

O diagnóstico da sífilis pode ser realizado pela visualização direta do treponema em campo escuro ou imunofluorescência direta, sendo tais técnicas indicadas na sífilis primária, lesões mucocutâneas e na sífilis congênita recente. A microscopia em campo escuro é o padrão ouro para o diagnóstico da sífilis recente, pois os testes sorológicos podem ser negativos neste estágio. ${ }^{18}$

As provas sorológicas são as mais utilizadas no diagnóstico da sífilis e dividem-se em: testes não treponêmicos, que são utilizados no diagnóstico e seguimento pós-tratamento, dos 
quais fazem parte o VDRL (Venereal disease research laboratory) e o RPR (Rapid plasma reagin), e os testes treponêmicos, válidos para confirmação da infecção, que incluem o FTA-ABS (Fluorescent treponemal antibody absorption) e TPHA (Treponema pallidum hemagglutination assay). Além destes, o imunoensaio enzimático (EIA) vem se tornando popular nos Estados Unidos como exame para rastreamento e poderá substituir os testes não treponêmicos para esse fim. É mais específico que o RPR e mais sensível que o FTA-ABS para infecções passadas. A técnica de PCR é pouco empregada na prática, porém pode ser aplicada, sobretudo, nas lesões de sífilis primária, congênita e neurossífilis. O Western-blot é outro teste ainda sem aplicação rotineira. ${ }^{19}$

Os testes não treponêmicos são testes de floculação quantitativa e seus títulos estão relacionados à atividade da doença e são utilizados no acompanhamento do tratamento. O VDRL é o mais utilizado para confirmação diagnóstica, pois apresenta boa sensibilidade e especificidade, podendo permanecer reagente mesmo após a cura da infecção (cicatriz sorológica), porém com queda progressiva das titulações.

Os testes treponêmicos são qualitativos e aferem a presença de anticorpos contra os antígenos de superfície do agente etiológico. Esses testes não se relacionam com a atividade da doença e não são úteis para detectar a reinfecção. Permanecem positivos por toda a vida e, quando negativos, excluem infecção prévia. ${ }^{20}$

O diagnóstico de sífilis na gestação em nosso país pode ser feito utilizando-se teste não treponêmico de floculação do tipo VDRL, para triagem. Em caso de positividade, recomenda-se a confirmação diagnóstica com teste treponêmico por aglutinação de partículas (TPHA) ou teste fluorescente por absorção de anticorpos (FTAAbs), entretanto a ausência desses não deve retardar o tratamento. Se o VDRL é reagente, e o teste treponêmico não reagente, trata-se de falso positivo, fato que ocorre em até $30 \%$ dos casos, após a confirmação por teste treponêmico. ${ }^{21}$ Pode-se utilizar também teste rápido por tiras de imunocromatografia, treponêmico, exame simples que pode ser realizado ambulatorialmente, com sangue total ou gota obtida da ponta do dedo, sendo vantajosa a sua utilização em lugares sem recursos laboratoriais, obtendo-se o diagnóstico em até 20 minutos. ${ }^{21}$

A introdução do teste rápido no arsenal diagnóstico para melhorar a cobertura de triagem para sífilis na gravidez, permitindo tratamento imediato, é sugestão na literatura internacional e já tem sido discutida para ser adotada em território nacional, à semelhança do que se faz com o teste rápido de HIV. Compõe a proposta de qualificação da atenção pré-natal dentro do projeto Rede Cegonha, do Ministério da Saúde. A portaria do Ministério da Saúde, de 30 de dezembro de 2011, estabelece a possibilidade da utilização de teste rápido para diagnóstico de sífilis na gestante e no parceiro, por profissionais capacitados, nas unidades de saúde, na etapa I do diagnóstico de sífilis. O teste rápido seria utilizado quando a gestante comparece para consulta pré-natal tardiamente ou quando não há acesso ao laboratório para realização do teste não-treponêmico (VDRL). Nestas situações, seria realizado o teste rápido e dada a conduta imediatamente, com aplicação da primeira dose de penicilina, se a amostra for reagente. Se possível, deve ser realizada a coleta de sangue por punção venosa para a etapa II. ${ }^{22,23}$

O Ministério da Saúde preconiza que durante a assistência pré-natal toda gestante seja submetida a pelo menos dois exames de VDRL, um por ocasião da primeira consulta e outro por volta da $28^{\text {a }}$ semana gestacional. Deve-se ainda realizar novo VDRL no momento do parto para garantir ao recém-nascido a possibilidade de tratamento precoce, caso a gestante não tenha sido tratada ou tenha se reinfectado após o tratamento. ${ }^{24}$

O diagnóstico diferencial da sífilis primária deve incluir herpes genital, cancro mole, linfogranuloma venéreo, donovanose, granuloma inguinal, erupções medicamentosas, carcinoma, infecções fúngicas superficiais e líquen plano. Na sífilis secundária deve-se fazer diagnóstico diferencial com pitiríase rósea, erupções medicamentosas, psoríase, líquen plano, escabiose, exantemas do sarampo e rubéola. ${ }^{25}$

\section{Tratamento}

O tratamento da gestante infectada pelo Treponema é igual ao de não gestantes e de acordo com o preconizado: penicilina benzatina IM na dose de 2.400.000 UI (1.200.000 UI em cada glúteo), com uma aplicação na sífilis primária, duas 
na sífilis secundária e três na sífilis terciária, com intervalos semanais entre as aplicações. Qualquer outro tratamento é considerado inadequado para a prevenção da sífilis congênita. O tratamento com penicilina somente é considerado eficaz, tanto para a mulher quanto para o feto, se administrado com mais de 30 dias antes do parto. ${ }^{26}$

A elevação de títulos do VDRL em quatro ou mais vezes (por exemplo, de 1:2 para 1:8) em relação ao último exame realizado, justifica novo tratamento. Verificar se o tratamento do parceiro foi realizado. Estes devem ser tratados mesmo quando apresentam VDRL negativo, com dose única de penicilina benzatina 2.400.000 UI IM; sendo o VDRL positivo, em qualquer titulação, o tratamento deve ser igual ao da sífilis terciária. A condição de parceiro não tratado caracteriza tratamento materno inadequado e, por conseguinte, a criança será considerada caso de sífilis congênita. ${ }^{27}$

No caso de alergia à penicilina, a gestante deverá ser dessensibilizada por meio da administração oral de penicilina $V$ em diluições gradativamente menores e em volumes inversamente maiores, devendo ser realizada em ambiente hospitalar para melhor manejo de possíveis reações. A administração da penicilina parenteral deverá ser feita somente 30 minutos após o término do processo de dessensibilização. Em casos de reações, a mesma deverá ser interrompida e deverá ser instituído um tratamento alternativo. O tratamento das gestantes alérgicas com estearato de eritromicina poderá levar à cura da gestante, mas não do feto. É feito na dose de 500 mg a cada 6 horas, por 15 dias na sífilis primária, secundária e latente precoce, ou durante 30 dias na sifilis latente tardia. Deve-se ter em mente a possibilidade de falência terapêutica nos casos de coinfecção pelo $\mathrm{HIV}^{28}$

Quando o tratamento é feito na fase secundária da doença, é maior a possibilidade de ocorrência da reação de Jarisch-Herxheimer, devido à liberação maciça de antígenos treponêmicos mortos na circulação. Os sintomas mais comuns são calafrios, febre, dor de cabeça e dores musculares, que, em geral, aparecem 4-6 horas após a administração da penicilina benzatina. Na gestante, tal reação pode levar à morte fetal, indicando necessidade de acompanhamento rigoroso no tratamento das portadoras de sífilis secundária com títulos altos de VDRL. O tratamento durante o pré-natal pode resultar em falha em até $14 \%$ dos casos e ainda há a possibilidade de reinfecção, caso o parceiro não tenha sido tratado simultaneamente. Por isso, o seguimento pós-tratamento deve ser realizado, segundo o Ministério da Saúde, com repetição da sorologia em 3, 6, 9, 12 e 18 meses. Considera-se cura quando há queda de três a quatro diluições no VDRL em relação à titulação inicial no prazo de um ano. ${ }^{29}$

Apesar de a sífilis apresentar recursos diagnósticos e terapêuticos simples e de baixo custo, seu controle na gestação mostra-se um desafio para profissionais de saúde e gestores. Este fato ocorre devido aos entraves para a realização do seu diagnóstico e tratamento, assim como, dificuldade de abordagem das doenças sexualmente transmissíveis, principalmente durante a gestação; parceiros sexuais que não são diagnosticados e/ou tratados e provavelmente pelo desconhecimento da magnitude desse agravo e dos danos que ele pode causar à saúde da mulher e do bebê pela população e até mesmo pelos profissionais de saúde. ${ }^{30}$

\section{Referências}

1. Avelleira JCR, Bottino G. Sífilis: diagnóstico, tratamento e controle. An Bras Dermatol. 2006;81(2):111-26.

2. Duarte G. Sífilis e a gravidez... e a história continua! Rev. Bras. Ginecol Obstet. 2012;34 (2):49-51. http://dx.doi.org/doi.org/10.1590/S010072032012000200001 .

3. Campos AL, Araújo MA, Melo SP, Gonçalves ML. Epidemiologia da sífilis gestacional em Fortaleza, Ceará, Brasil: um agravo sem controle. Cad. Saude Publica. 2010; 26(9):1747-55. http://dx.doi.org/doi. org/10.1590/S0102-311X2010000900008.

4. Rodrigues CS, Guimarães MD, César CC. Missed opportunities for congenital syphilis and HIV perinatal transmission prevention. Rev Saude Publica. 2008;42(5):851-8.

5. Krakauer Y, Pariente G, Sergienko R, Wiznitzer A, Sheiner E. Perinatal outcome in cases of latent syphilis during pregnancy. Int J Gynaecol Obstet. 2012;118(1):15-7 http://dx.doi.org/10.1016/j. ijgo.2012.01.026.

6. Brasil, Ministério da Saúde. Coordenação de Informações Estratégicas - CIE. Departamento de DST, Aids e Hepatites Virais. Boletim Epidemiológico Aids e DST, 2013. Brasília: 2013.http://www.aids.gov. br/publicacao/2013/boletim-epidemiologico-aids-edst-2013 (Acesso em: 09 fev 2014). 
7. Brasil, Ministério da Saúde. Secretaria de Vigilância em Saúde. Departamento de DST, Aids e Hepatites Virais. Boletim Epidemiológico-Sífilis, 2012. Brasília: Ministério da Saúde; 2012. http://www.aids.gov. br/publicacao/2012/boletim-epidemiologico-desifilis-2012 (Acesso em: 09 fev 2014).

8. Fenton KA, Breban R, Vardavas R, Okano JT, Martin $\mathrm{T}$, Aral S, et al. Infectious syphilis in high-income settings in the $21^{\text {st }}$ century. Lancet Infect Dis. 2008;8(4):244-53. http://dx.doi.org/10.1016/S14733099(08)70065-3.

9. Kent ME, Romanelli F. Reexamining syphilis: an update on epidemiology, clinical manifestations, and management. Ann Pharmacother. 2008;42(2):226-36. http://dx.doi.org/10.1345/aph.1K086.

10. Oliveira EVL, Filho JAR, Monteiro AA, Pozzeti EMO, Antonio JR. Sífilis secundária com acometimento pulmonar. An. Bras. Dermatol. 2007;82(2):163-67. http:// dx.doi.org/10.1590/S0365-05962007000200008.

11. Sukthankar A. Syphilis. Medicine. 2010;38(5):263-6. http://dx.doi.org/10.1016/j.mpmed.2010.01.016.

12. Thomas DD, Navab M, Haake DA, Fogelman AM, Miller JN, Lovett MA. Treponema pallidum invades intercellular junctions of endothelial cell monolayers. Proc Natl Acad Sci U.S.A.1988;85:3608-3612.

13. Riley BS, Oppenheimer-Marks N, Hansen EJ, Radolf JD, Norgard MV. Virulent Treponema pallidum activates human vascular endothelial cells. J Infect Dis. 1992;165(3): 484-93. http://dx.doi.org/10.1093/ infdis/165.3.484

14. Nascimento MI, Cunha AA, Guimarães EV, Alvarez FS, Oliveira SR, Villas Bôas EL. Gestações complicadas por sífilis materna e óbito fetal. Rev Bras Ginecol Obstet. 2012;34(2):56-62.

15. Costa MC, Demarch EB, Azulay DR, Périssé ARS, Dias MFRG, Nery JAC. Doenças sexualmente transmissíveis na gestação: uma síntese de particularidades. An Bras Dermatol. 2010;85(6):767-85. http:// dx.doi.org/10.1590/S0365-05962010000600002.

16. Hawkes S, Matin N, Broutet N, Low N. Effectiveness of interventions to improve screening for syphilis in pregnancy: a systematic review and meta-analysis. Lancet Infect Dis 2011;11(9):684-91. http://dx.doi. org/10.1016/S1473-3099(11)70104-9.

17. O'Connor M, Kleinman S, Goff M. Syphilis in Pregnancy. Journal of Midwifery \& Women's Health.2008;53(3):e17-e21.

18. Belda Jr W, Shiratsu R, Pinto V. Abordagem nas doenças sexualmente transmissíveis. An Bras Dermatol. 2009;84(2):151-9. http://dx.doi.org/10.1590/S036505962009000200008.
19. Rotta O. Diagnóstico sorológico da sífilis. An Bras Dermatol. [periódico na Internet] 2005;80(3):299302. http://dx.doi.org/10.1590/S036505962005000300014. [Acesso em 23 maio 2014]

20. Chufalo JE, Maciel ACG, Espíndola LN. Sífilis Congênita. Femina. 2006;34(10):701-10.

21. Amaral E. Sífilis na gravidez e óbito fetal: de volta para o futuro. Rev Bras Ginecol Obstet. 2012; 34(2): 52-5. http://dx.doi.org/10.1590/S010072032012000200002 .

22. Brasil. Ministério da Saúde. Departamento de Ações Programáticas Estratégicas. Área Técnica de Saúde da Mulher [Internet]. Diretrizes gerais e operacionais da Rede Cegonha. 2011 [Acesso em 1 jan 2012].

23. Brasil. Ministério da Saúde [Internet]. Portaria $N^{0}$ 3.242, de 30 de dezembro de 2011. Dispõe sobre o Fluxograma Laboratorial da Sífilis e a utilização de testes rápidos para triagem da sífilis em situações especiais e apresenta outras recomendações. 2011 [Acesso em 2 jan 2012].

24. Campos AL, Araújo MA, Melo SP, Andrade RF, Gonçalves ML. Syphilis in parturients: aspects related to the sex partner. Rev Bras Ginecol Obstet. 2012;34(9):397-402. http://dx.doi.org/10.1590/S010072032012000900002.

25. Júnior MFQS. Sífilis. In: Veronesi R, Focaccia R. Tratado de infectologia. $3^{\text {a }}$.ed. São Paulo: Atheneu; 2005. p.1263-72.

26. Duarte G. Doenças sexualmente transmissíveis e gravidez. In: Linhares IM, Duarte G, Giraldo PC, Bagnoli VR (eds.) Manual de Orientação, DST/AIDSFederação Brasileira das Associações de Ginecologia e Obstetrícia (FEBRASGO). São Paulo: Editora Ponto; 2004. p.118-41.

27. Saraceni V, Leal MC. Avaliação da efetividade das campanhas para eliminação da sífilis congênita na redução da morbimortalidade perinatal: Município do Rio de Janeiro, 1999-2000. Cad. Saúde Pública. 2003;19:1341-9.

28. Brasil. Ministério da Saúde. Secretaria de Vigilância em Saúde. Programa Nacional de DST/AIDS. Manual de Controle das Doenças Sexualmente Transmissíveis. 4 ed. Brasília: Ministério da Saúde, 2006.

29. Manela-Azulay M, Azulay DR. Treponematoses, sífilis. In: Azulay RD, Azulay DR, Azulay-Abulafia L (eds.) Dermatologia. 5 ed. Rio de Janeiro: Guanabara Koogan; 2008, p. 349-66.

30. Domingues RMSM, Saraceni V, Hartz ZMA, Leal MC. Sífilis congênita: evento sentinela da qualidade da assistência pré-natal. Rev Saude Publica. 2013;47(1):147-57 


\section{Alessandra B. A. Damasceno}

Divisão de Obstetrícia. Hospital Maternidade Carmela Dutra. Secretaria Municipal de Saúde e Defesa Civil do Rio de Janeiro. Rio de Janeiro, RJ, Brasil.

\section{Denise L. M. Monteiro}

Disciplina de Obstetrícia. Departamento de Ginecologia e Obstetrícia. Centro Universitário Serra dos Órgãos (UNIFESO). Teresópolis, RJ, Brasil.

Disciplina de Obstetrícia. Departamento de Ginecologia e Obstetrícia. Faculdade de Ciências Médicas da Universidade do Estado do Rio de Janeiro. Rio de Janeiro, RJ, Brasil.

\section{Luiza B. Rodrigues}

Iniciação Científica, FAPERJ. Faculdade de Ciências Médicas. Universidade do Estado do Rio de Janeiro. Rio de Janeiro, RJ, Brasil.

\section{Danielle B. S. Barmpas}

Programa de Pós-graduação em Ciências Médicas. Faculdade de Ciências Médicas. Universidade do Estado do Rio de Janeiro. Rio de Janeiro, RJ, Brasil.

\section{Luciane R. P. Cerqueira}

Programa de Pós-graduação em Ciências Disciplina de Obstetrícia. Departamento Ginecologia e Obstetrícia. Faculdade de Ciências Médicas. Universidade do Estado do Rio de Janeiro. Rio de Janeiro, RJ, Brasil.

\section{Alexandre J. B. Trajano}

Departamento de Ginecologia e Obstetrícia. Faculdade de Ciências Médicas. Universidade do Estado do Rio de Janeiro. Rio de Janeiro, RJ, Brasil.

Departamento de Ginecologia e Obstetrícia. Faculdade de Medicina. Universidade Unigranrio. Rio de Janeiro, RJ, Brasil. 